\title{
OncoLnc: Linking TCGA survival data to mRNAs, miRNAs, and IncRNAs
}

Jordan Anaya

Oncolnc is a tool for interactively exploring survival correlations, and for downloading clinical data coupled to expression data for mRNAs, miRNAs, or IncRNAs. OncoLnc contains survival data for 8,647 patients from 21 cancer studies performed by The Cancer Genome Atlas (TCGA), along with RNA-SEQ expression for mRNAs and miRNAs from TCGA, and IncRNA expression from MiTranscriptome beta. Storing this data gives users the ability to separate patients by gene expression, and then create publication-quality Kaplan-Meier plots or download the data for further analyses. OncoLnc also stores precomputed survival analyses, allowing users to quickly explore survival correlations for up to 21 cancers in a single click. This resource allows researchers studying a specific gene to quickly investigate if it may have a role in cancer, and the supporting data allows researchers studying a specific cancer to identify the mRNAs, miRNAs, and IncRNAs most correlated with survival, and researchers looking for a novel IncRNA involved with cancer lists of potential candidates. OncoLnc is available at http://www.oncolnc.org. 


\section{OncoLnc: Linking TCGA survival data to mRNAs, miRNAs, and 2 IncRNAs}

3 Jordan Anaya ${ }^{1}$

4 10mnesres.com, email: omnesresnetwork@gmail.com, twitter: @omnesresnetwork

5 Corresponding Author:

6 Jordan Anaya

7 Charlottesville, VA, US

8 email address: omnesresnetwork@gmail.com

9

10

11

12

13

14

15

16

17

18

19

20

21

22

23

24

25 


\section{Abstract}

OncoLnc is a tool for interactively exploring survival correlations, and for downloading clinical data coupled to expression data for mRNAs, miRNAs, or lncRNAs. OncoLnc contains survival data for 8,647 patients from 21 cancer studies performed by The Cancer Genome Atlas (TCGA), along with RNA-SEQ expression for mRNAs and miRNAs from TCGA, and lncRNA expression from MiTranscriptome beta. Storing this data gives users the ability to separate patients by gene expression, and then create publication-quality Kaplan-Meier plots or download the data for further analyses. OncoLnc also stores precomputed survival analyses, allowing users to quickly explore survival correlations for up to 21 cancers in a single click. This resource allows researchers studying a specific gene to quickly investigate if it may have a role in cancer, and the supporting data allows researchers studying a specific cancer to identify the mRNAs, miRNAs, and lncRNAs most correlated with survival, and researchers looking for a novel lncRNA involved with cancer lists of potential candidates. OncoLnc is available at http://www.oncolnc.org.

\section{Main article text}

\section{Introduction}

The Cancer Genome Atlas (TCGA) provides researchers with unprecedented amounts of molecular data along with clinical and histopathological information (http://cancergenome.nih.gov/). This data set has not only led to increases in our understanding of cancer (Ciriello et al. 2013; Hoadley et al. 2014), but its scale has also allowed for previously impossible projects such as a comprehensive cataloguing of the human transcriptome (Han et al. 2014; Iyer et al. 2015). However, the size and complexity of this unique data set makes it difficult for cancer researchers to access and fully utilize.

Multiple resources exist to help researchers download or explore TCGA data (Cerami et al. 2012; Gyorffy et al. 2013; Koch et al. 2015). However, a tool does not exist that focuses on survival analyses with TCGA data. Although cBioPortal (http://www.cbioportal.org/) does allow for simple Kaplan-Meier analyses for a range of TCGA cancer studies, it does not allow for more rigorous analyses with Cox regression. More importantly, the p-value obtained from a single analysis can be misleading, and it may be more informative to consider the relative strength of the correlation (Anaya et al. 2016).

Furthermore, current tools for survival analyses only allow users to view the results from one cancer at a time. This makes it difficult for a researcher to perform a comprehensive survival study with their gene of interest, and makes it possible that researchers will miss an interesting correlation. Current tools also do not allow for generation of Kaplan-Meier plots with user defined upper and lower groups, and do not allow for simple download of the survival data coupled to the expression data. 
62 It is also important to note that the gene names used in TCGA data are outdated. While many

63

64

65

online portals use current mRNA definitions, there is not an online data portal that uses modern miRNA definitions. This is because the TCGA Tier 3 read counts for the 5p and $3 p$ arms are aggregated into one count for the stem-loop, which makes it difficult for researchers who want to obtain information for a specific mature miRNA.

In addition, although the role of long noncoding RNAs (lncRNAs) in cancer is beginning to be appreciated (Yarmishyn \& Kurochkin 2015), the Tier 3 TCGA mRNA files contain expression data for only the limited number of lncRNAs that were known at the initiation of the TCGA project. As a result, tools for exploring TCGA data will not contain many lncRNAs currently being studied. Although a platform has already been developed to fill this gap (Li et al. 2015), to help the scientific community study lncRNAs OncoLnc incorporates analyses and data for MiTranscriptome beta lncRNAs, http://mitranscriptome.org/, in addition to Tier 3 TCGA mRNAs and miRNAs.

\section{Materials and methods}

\section{Code, files, and software}

All of the code necessary to reproduce Tables 1, 2, 3, S1, S2, and S3, and therefore the data in OncoLnc, along with a limited amount of raw data and intermediate files is located at https://github.com/OmnesRes/onco_lnc. The rest of the raw data can be downloaded from https://tcga-data.nci.nih.gov/tcga/ and http://mitranscriptome.org/. This code was run with Python 2.7.5, NumPy 1.7.1, and rpy2 2.5.6, and can require upwards of 6GB of RAM. OncoLnc runs on Django 1.8.2, Python 2.7, matplotlib 1.2.1, NumPy 1.7.1, rpy2 2.5.6, uses the SQLite3 database engine, and utilizes Bootstrap CSS and JavaScript, and Font Awesome icons.

\section{Cox models}

Multivariate Cox regressions were performed with the coxph function from the $\mathrm{R}$ survival library. For each cancer and data type an attempt was made to construct a model with gene expression, sex, age, and grade or histology as multivariates. However, not every cancer contained complete grade information, and some cancers did not contain different grades. As a result, grade was not able to be included in every model. Cancers which have grade in the model are BLCA, BRCA, CESC, ESCA, HNSC, KIRC, LGG, LIHC, OV, PAAD, STAD, and UCEC. Cancers with grade missing from the model include COAD, GBM, KIRP, LAML, LUAD, LUSC, READ, SARC, and SKCM. When grade is available each grade is listed as a separate term in the models and is either 1 or 0 . Similarly, sex is also listed in the models as 1 or 0 . Gene expression is inverse normal transformed before inclusion into the models, except for the GBM miRNA data, which was normalized microarray data. Age is listed in the models as is. The multivariate model run for each cancer and each data type is listed at the top of Tables S1, S2, and S3, and the code for running all of the Cox regressions is present in the GitHub repository: https://github.com/OmnesRes/onco_lnc. 
All the clinical data was downloaded from https://tcga-data.nci.nih.gov/tcga/ January 5th and 6th, 2016. Cancers were chosen for the study based on the quality and amount of overall survival data. It is possible for a cancer such as PRAD to have a large amount of survival information, but a low number of events (deaths), making a survival analysis difficult. The Tier $3 \mathrm{mRNA}$ and miRNA data was also downloaded from https://tcga-data.nci.nih.gov/tcga/, while the MiTranscriptome data was downloaded from http://mitranscriptome.org/. Definitions for miRNAs were downloaded from http://www.mirbase.org/.

For each cancer, only patients who contained all the necessary clinical information were included in the analysis. In addition, patients had to have a follow up time or time to death greater than 0 days. For each cancer, only genes which met an expression cutoff were included in the analysis (see below for more details). In general only primary solid tumors were included in analyses, and this is implemented by only using samples with "01" in the patient barcode. The exceptions are LAML, which is a blood derived cancer, and therefore has the designation "03", and SKCM, which contains primarily metastatic tumors, and therefore designations " 01 " and " 06 " were allowed for SKCM analyses. It is possible for a patient to have more than one sequencing file, and in these cases the counts were averaged.

\section{Results}

\section{Overview of OncoLnc}

OncoLnc stores over 400,000 analyses, which includes Cox regression results as well as mean and median expression of each gene. For the Cox regression results, in addition to p-values, OncoLnc stores the rank of the correlation. Different cancers contain very different $p$-value distributions (Anaya et al. 2016; Yang et al. 2014), and it is unclear what causes this difference. As a result, using one p-value cutoff across cancers is not possible, and the rank of the correlation is a simple way to measure the relative strength of the correlation. The rank is calculated per cancer, per data type. Tables 1-3 contain information about how many genes there are for each cancer and each data type.

The mRNA and miRNA identifiers used by TCGA are out of date, and the identifiers in OncoLnc have been manually curated using NCBI Gene: http://www.ncbi.nlm.nih.gov/gene, and recent miRBase definitions: http://www.mirbase.org/. Over 2,000 mRNA symbols were updated, and these are listed in Table S4. Genes which have had their Entrez Gene ID removed from NCBI Gene, or could not be confidently mapped to a single identifier, are not included in OncoLnc but are still included in Table S1.

Using OncoLnc is very straightforward. The preferred method of using OncoLnc is to submit a gene at the home page, and this submission is not case sensitive. If a user submits a gene not in the database they will be notified and provided with links to all the possible gene names and IDs. Submission of a valid gene identifier will return correlation results for up to 21 cancers for mRNAs and miRNAs, or 18 cancers for MiTranscriptome beta lncRNAs (Fig. 1). If a gene does 
136

137

138

139

140

141

142

143

144

145

146

147

148

149

150

151

152

153

154

155

156

157

158

159

160

161

162

163

164

165

166

167

168

169

170

171

not meet the expression cutoff for the analysis, it will not be present in the database, and therefore a user may receive less than the maximum possible number of results. For users using OncoLnc on smaller devices, it is possible to perform a single cancer search. The link for this search is on the home page, and the user must submit the TCGA cancer abbreviation along with the gene of interest.

At the results page is a link to perform a Kaplan-Meier analysis for each cancer (Fig. 1). The user will be asked how they would like to divide the patients. Patients can be split into any nonoverlapping upper and lower slices, for example upper 25 percent and lower 25 percent. Upon submission users will be presented with a PNG Kaplan-Meier plot, a logrank p-value for the analysis, and text boxes with the data that was plotted (Fig. 2). If a user simply wants all the data for that cancer and that gene, the user can submit 100 for "Lower Percentile", and 0 for "Upper Percentile".

Users then have the option to either go to a PDF of the Kaplan-Meier plot, or download a CSV file of the data plotted. In both cases the file name will be the cancer, gene ID, lower percentile, upper percentile, separated by underscores. Gene ID had to be used instead of gene name because there are multiple HUGO gene symbol conflicts between TCGA Tier 3 mRNAs and MiTranscriptome beta, as well as between TCGA mRNA HUGO gene symbols and updated mRNA HUGO gene symbols. In the case that a user performs a search for a name with a conflict, OncoLnc presents a warning message and instructs the user how to proceed.

\section{mRNAs}

Table 1 contains information about the patients for each Tier 3 mRNA study included in OncoLnc, and how many gene analyses are present in OncoLnc for each study. Tier 3 RNASeqV2 was used for all 21 cancers, and expression was taken from the "rsem.genes.normalized_results" files. As a result, the expression data in OncoLnc for Tier 3 mRNAs is in normalized RSEM values. Table 1 contains different numbers of genes for the different cancers because an expression cutoff was used to determine if a gene would be included in the analysis. For mRNAs this cutoff was a median expression greater than 1 RSEM, and less than a fourth of the patients with an expression of 0 .

The results of every Tier 3 mRNA Cox regression performed are included in Table S1. The Tier 3 expression files contain both a HUGO gene symbol and Entrez Gene ID for each gene, but these IDs and gene symbols are not current. To update the gene symbols I downloaded every human gene from NCBI Gene, and updated any symbol for which the Entrez Gene ID was still current. For genes that had deleted or changed Entrez Gene IDs I had to manually curate the Gene IDs and gene symbols. Genes which I could not confidently assign to a modern ID are not included in OncoLnc, but are still included in Table S1. Table S1 includes the original TCGA IDs and symbols along with the updated names and symbols, and Table S4 lists genes which had 
172 either the symbol or ID changed. OncoLnc allows users to search mRNAs using either an

173 updated HUGO gene symbol or Entrez Gene ID.

174

175

176

177

178

179

180

181

182

183

184

185

186

187

188

189

190

191

192

193

194

195

196

197

198

199

200

201

202

203

204

205

206

207

208

\section{miRNAs}

Table 2 contains information about the patients for each Tier 3 miRNA study included in OncoLnc, and how many gene analyses are present in OncoLnc for each study. Tier 3 miRNASeq was used for every cancer except GBM, which only had microarray data available. The results of every Cox regression performed are included in Table S2. Many of the miRBase IDs, or possibly read counts, present in Table S2 and OncoLnc will be different from the IDs and read counts in TCGA data files and available at other data portals for TCGA data. This is because I went through each expression file and updated the IDs and read counts.

The "isoform.quantification" files contain both miRBase IDs as well accession numbers. In these files the $5 p$ and $3 p$ arms of miRNAs are referred to with the same ID, for example hsa-let$7 b-5 p$ and hsa-let-7b-3p would both be listed as hsa-let-7b. In order to update the names and read counts for the Tier 3 miRNAs I used the read counts assigned to each accession number to obtain reads per million miRNAs mapped for each accession number, and updated the ID with the current miRBase ID. When an accession number was not available I used the genomic coordinates provided to identify the accession number, and therefore ID. GBM names were updated using the "aliases" file from the miRBase FTP site, and if an alias could not be confidently identified the miRNA was not included in OncoLnc, but is still in Table S2.

As a result, all expression values in Table S2 and in OncoLnc are reads per million miRNA mapped for every cancer except GBM, which are microarray normalized values. The numbers of miRNAs in Table 2 differ because the miRNA may not have been in the expression files for that cancer, or may not have met the expression cutoff. An expression cutoff of a median of .5 reads per million miRNA mapped, and less than one fourth of the patients with 0 expression was used. OncoLnc allows users to search for miRNAs with either a miRBase version 21 mature accession number or ID.

\section{IncRNAs}

Table 3 contains information about the patients for each MiTranscriptome beta lncRNA analysis, along with how many lncRNAs are included in OncoLnc for each cancer. Normalized lncRNA counts were downloaded from http://mitranscriptome.org/, and these were mapped to patient barcodes using the library information provided. MiTranscriptome beta contains over 8,000 of the most differentially expressed lncRNAs in the entire MiTranscriptome dataset, but the actual number of lncRNAs in OncoLnc for each cancer is far fewer due to the expression cutoff used: a median of . 1 normalized counts, and less than a fourth of patients with 0 expression. Table S3 contains every lncRNA Cox regression performed, and these are all included in OncoLnc. OncoLnc allows users to search for MiTranscriptome beta lncRNAs using either a name or transcript ID. 


\section{Discussion}

210 Depending on the researcher, OncoLnc should be used in different ways. If a researcher is

211

212

213

214

215

216

217

218

219

220

221

222

223

224

225

226

227

228

229

230

231

232

233

234

235

236

237

238

239

240

241

242

243

244

245

246 studying a specific gene and looking for a cancer association, they should go to http://www.oncolnc.org and perform a search with their gene of interest. Instead of focusing on p-values, I would focus more on the rank of the correlations for the different cancers, and also on the sign of the Cox coefficients. A positive Cox coefficient indicates high expression of the gene increases the risk of death, while a negative Cox coefficient indicates the opposite. A gene with a high rank in multiple cancers (indicated by a low number, 1 being the best), and Cox coefficients with the same sign could be very interesting. It is also important to look at the level of expression of the gene. Different genes obviously require different levels of expression to exert their effects, but genes with expression near 0 should be dealt with caution. In addition, users can investigate the range of expression of the gene at the Kaplan-Meier plotting page. Genes that have large fold increases from the low expression to high expression group could be interesting candidates.

A researcher studying a specific cancer should download Tables S1, S2, and S3 to see which mRNAs, miRNAs, and lncRNAs are most correlated to survival for their cancer. Once they identify some genes of interest they can go to http://www.oncolnc.org to perform further analyses such as checking the range of expression of the gene, or if it is associated with survival in other cancers. Similarly, bioinformaticians looking to perform large scale analyses of prognostic genes can use these tables as a starting point, or if a user wants to change the Cox models they can use the GitHub code to alter the models.

The importance of the ability to perform survival correlations with lncRNAs must be emphasized. There are multiple techniques for identifying protein coding genes that are involved in cancer because mutations that occur in protein coding genes can result in missense mutations, and methods have been developed for identifying which of these mutations are drivers as opposed to simply passengers (Carter et al. 2009; Kaminker et al. 2007; Youn \& Simon 2011). In contrast, because it is unclear how mutations will affect lncRNA function, methods to identify lncRNAs involved in cancer must rely on lncRNA expression. As a result, OncoLnc is one of the few resources available for finding lncRNAs involved in cancer, and if a lncRNA researcher is searching for a novel lncRNA to study, Table S3 would be a good place to start.

Compared to microarrays, RNA-SEQ is a relatively unproven technology for identifying prognostic genes. However, I previously performed a pan-cancer analysis of prognostic genes that used RNA-SEQ data and provided consistent groupings of cancers and identified interesting cancer biology (Anaya et al. 2016). For example, in my analysis EGF signaling was strongly associated with survival in LUSC, and EGFR inhibitors have been shown to be effective in LUSC patients despite EGFR mutations being rare in this cancer (Chiu et al. 2014). Although examples such as this are assuring, when using OncoLnc it is important to remember that the correlations observed, regardless of p-value, are still only correlations. Perhaps the largest 
247 limitation of OncoLnc is that the Cox models do not account for intra-cancer subtypes. For

248 example, GBM and BRCA both have well-established subtypes (Brennan et al. 2013; Perou et al.

249 2000). If the expression of a gene correlates with cancer subtypes, and those subtypes correlate

250 with survival, subtype would be a confounding variable. As subtype definitions for the different

251 cancers improve a future version of OncoLnc may be able to incorporate the subtypes in the Cox

252 models.

253 An analysis is only as good as the data available, and the Tier 3 TCGA RNA-SEQ analyses were 254 performed with outdated software and transcript information. There have been some attempts to

255 reanalyze both the TCGA mRNA RNA-SEQ data and miRNA-SEQ data (Kuo et al. 2015;

256 Rahman et al. 2015). In the event that TCGA or the scientific community releases a gold

257 standard analysis of TCGA data, a future version of OncoLnc could incorporate this data.

258 Current data portals for TCGA data only allow users to view the results for one cancer at a time, 259 may or may not offer Cox regression results, do not allow for complete control over separating 260 patients during Kaplan-Meier analysis, and do not allow for download of the data used in the 261 analysis. To my knowledge OncoLnc is the only online resource for TCGA data that includes 262 these features, is the only resource that uses modern gene definitions for TCGA mRNA and 263 miRNA data, and is the only resource for survival analysis of MiTranscriptome beta lncRNAs. 264 In addition, current methods for survival analysis rely on a p-value cutoff of .05 for significance, 265 which may lead to either the study of genes not actually correlated with survival or missing 266 genes that are correlated with survival depending on the cancer. By storing the results of the 267 correlation for every gene, OncoLnc can provide a context for the significance of a correlation.

268 As a result, used correctly OncoLnc can not only increase the sensitivity of finding genes 269 involved in cancer, but also the specificity. This combination of ease of use, results for complex 270 analyses, and tools for exploring and downloading data make OncoLnc an invaluable resource 271 for cancer researchers.

\section{Additional Information and Declarations}

\section{Competing Interests}

274 I offer services at omnesres.com which may involve web development or analysis of TCGA data.

\section{Author Contributions}

276 I conceived the project, downloaded and analyzed all data, developed OncoLnc, wrote the

277 manuscript, and maintain the GitHub repository and any associated material at omnesres.com.

\section{Funding}

279 This project was not funded. 
281 This project was made possible by data generated by the TCGA Research Network:

282 http://cancergenome.nih.gov/.

\section{References}

284

285

286

287

288

289

290

291

292

293

294

295

296

297

298

299

300

301

302

303

304

305

306

307

308

309

310

311

312

313

314

315

316

317

318

319

320

321

322

323

324

325

326

Anaya J, Reon B, Chen W, Bekiranov S, and Dutta A. 2016. A pan-cancer analysis of prognostic genes. PeerJ 3:e1499. 10.7717/peerj.1499

Brennan CW, Verhaak RG, McKenna A, Campos B, Noushmehr H, Salama SR, Zheng S, Chakravarty D, Sanborn JZ, Berman SH, Beroukhim R, Bernard B, Wu CJ, Genovese G, Shmulevich I, BarnholtzSloan J, Zou L, Vegesna R, Shukla SA, Ciriello G, Yung WK, Zhang W, Sougnez C, Mikkelsen T, Aldape K, Bigner DD, Van Meir EG, Prados M, Sloan A, Black KL, Eschbacher J, Finocchiaro G, Friedman W, Andrews DW, Guha A, lacocca M, O'Neill BP, Foltz G, Myers J, Weisenberger DJ, Penny R, Kucherlapati R, Perou CM, Hayes DN, Gibbs R, Marra M, Mills GB, Lander E, Spellman P, Wilson R, Sander C, Weinstein J, Meyerson M, Gabriel S, Laird PW, Haussler D, Getz G, Chin L, and Network TR. 2013. The somatic genomic landscape of glioblastoma. Cell 155:462-477. 10.1016/j.cell.2013.09.034

Carter H, Chen S, Isik L, Tyekucheva S, Velculescu VE, Kinzler KW, Vogelstein B, and Karchin R. 2009. Cancer-specific high-throughput annotation of somatic mutations: computational prediction of driver missense mutations. Cancer Res 69:6660-6667. 10.1158/0008-5472.can-09-1133

Cerami E, Gao J, Dogrusoz U, Gross BE, Sumer SO, Aksoy BA, Jacobsen A, Byrne CJ, Heuer ML, Larsson E, Antipin Y, Reva B, Goldberg AP, Sander C, and Schultz N. 2012. The cBio cancer genomics portal: an open platform for exploring multidimensional cancer genomics data. Cancer Discov 2:401404. 10.1158/2159-8290.cd-12-0095

Chiu CH, Chou TY, Chiang CL, and Tsai CM. 2014. Should EGFR mutations be tested in advanced lung squamous cell carcinomas to guide frontline treatment? Cancer Chemother Pharmacol 74:661665. 10.1007/s00280-014-2536-3

Ciriello G, Miller ML, Aksoy BA, Senbabaoglu Y, Schultz N, and Sander C. 2013. Emerging landscape of oncogenic signatures across human cancers. Nat Genet 45:1127-1133. 10.1038/ng.2762

Gyorffy B, Surowiak P, Budczies J, and Lanczky A. 2013. Online survival analysis software to assess the prognostic value of biomarkers using transcriptomic data in non-small-cell lung cancer. PLoS One 8:e82241. 10.1371/journal.pone.0082241

Han L, Yuan Y, Zheng S, Yang Y, Li J, Edgerton ME, Diao L, Xu Y, Verhaak RG, and Liang H. 2014. The PanCancer analysis of pseudogene expression reveals biologically and clinically relevant tumour subtypes. Nat Commun 5:3963. 10.1038/ncomms4963

Hoadley KA, Yau C, Wolf DM, Cherniack AD, Tamborero D, Ng S, Leiserson MD, Niu B, McLellan MD, Uzunangelov V, Zhang J, Kandoth C, Akbani R, Shen H, Omberg L, Chu A, Margolin AA, Van't Veer LJ, Lopez-Bigas N, Laird PW, Raphael BJ, Ding L, Robertson AG, Byers LA, Mills GB, Weinstein JN, Van Waes C, Chen Z, Collisson EA, Cancer Genome Atlas Research N, Benz CC, Perou CM, and Stuart JM. 2014. Multiplatform analysis of 12 cancer types reveals molecular classification within and across tissues of origin. Cell 158:929-944. 10.1016/j.cell.2014.06.049

Iyer MK, Niknafs YS, Malik R, Singhal U, Sahu A, Hosono Y, Barrette TR, Prensner JR, Evans JR, Zhao S, Poliakov A, Cao X, Dhanasekaran SM, Wu YM, Robinson DR, Beer DG, Feng FY, Iyer HK, and Chinnaiyan AM. 2015. The landscape of long noncoding RNAs in the human transcriptome. Nat Genet 47:199-208. 10.1038/ng.3192

Kaminker JS, Zhang Y, Watanabe C, and Zhang Z. 2007. CanPredict: a computational tool for predicting cancer-associated missense mutations. Nucleic Acids Res 35:W595-598. 10.1093/nar/gkm405

Koch A, De Meyer T, Jeschke J, and Van Criekinge W. 2015. MEXPRESS: visualizing expression, DNA methylation and clinical TCGA data. BMC Genomics 16:636. 10.1186/s12864-015-1847-z 
327
Kuo WT, Su MW, Lee YL, Chen CH, Wu CW, Fang WL, Huang KH, and Lin WC. 2015. Bioinformatic Interrogation of 5p-arm and 3p-arm Specific miRNA Expression Using TCGA Datasets. J Clin Med 4:1798-1814. 10.3390/jcm4091798

Li J, Han L, Roebuck P, Diao L, Liu L, Yuan Y, Weinstein JN, and Liang H. 2015. TANRIC: An Interactive Open Platform to Explore the Function of IncRNAs in Cancer. Cancer Res 75:3728-3737. 10.1158/0008-5472.can-15-0273

Perou CM, Sorlie T, Eisen MB, van de Rijn M, Jeffrey SS, Rees CA, Pollack JR, Ross DT, Johnsen H, Akslen LA, Fluge O, Pergamenschikov A, Williams C, Zhu SX, Lonning PE, Borresen-Dale AL, Brown PO, and Botstein D. 2000. Molecular portraits of human breast tumours. Nature 406:747-752. $10.1038 / 35021093$

Rahman M, Jackson LK, Johnson WE, Li DY, Bild AH, and Piccolo SR. 2015. Alternative preprocessing of RNA-Sequencing data in The Cancer Genome Atlas leads to improved analysis results. Bioinformatics 31:3666-3672. 10.1093/bioinformatics/btv377

Yang Y, Han L, Yuan Y, Li J, Hei N, and Liang H. 2014. Gene co-expression network analysis reveals common system-level properties of prognostic genes across cancer types. Nat Commun 5:3231. 10.1038/ncomms4231

Yarmishyn AA, and Kurochkin IV. 2015. Long noncoding RNAs: a potential novel class of cancer biomarkers. Front Genet 6:145. 10.3389/fgene.2015.00145

Youn A, and Simon R. 2011. Identifying cancer driver genes in tumor genome sequencing studies. Bioinformatics 27:175-181. 10.1093/bioinformatics/btq630 


\section{Table $\mathbf{1}$ (on next page)}

Characteristics of the Tier 3 mRNA datasets in OncoLnc

Age at diagnosis is in years, and is an average. The events indicate the number of deaths in the dataset. Median survival is in days and could not be calculated for COAD, KIRP, OV, READ, and UCEC. 


$\begin{array}{lrlrrrr} & & & & & & \text { Genes } \\ \text { Cancer } & \text { Patients } & \text { Male/Female } & \begin{array}{r}\text { Age at } \\ \text { Diagnosis }\end{array} & \text { Events } & \begin{array}{r}\text { Median } \\ \text { Survival }\end{array} & \begin{array}{c}\text { incolnc } \\ \text { BLCA }\end{array} \\ \text { BRCA } & 1006 & 296 / 107 & 68.03 & 177 & 1008 & 16339 \\ \text { CESC } & 264 & 0 / 264 & 58.34 & 135 & 3941 & 16602 \\ \text { COAD } & 440 & 235 / 205 & 48.23 & 59 & 4086 & 16330 \\ \text { ESCA } & 144 & 126 / 18 & 66.58 & 85 & \text { NA } & 16378 \\ \text { GBM } & 152 & 99 / 53 & 60.51 & 59 & 801 & 16790 \\ \text { HNSC } & 497 & 364 / 133 & 59.84 & 27 & 1426 & 16783 \\ \text { KIRC } & 523 & 341 / 182 & 61.24 & 207 & 1732 & 16614 \\ \text { KIRP } & 285 & 210 / 75 & 60.56 & 167 & 2764 & 16638 \\ \text { LAML } & 151 & 81 / 70 & 61.45 & 44 & \text { NA } & 16399 \\ \text { LGG } & 510 & 282 / 228 & 54.40 & 92 & 577 & 15227 \\ \text { LIHC } & 360 & 244 / 116 & 43.02 & 124 & 2835 & 16781 \\ \text { LUAD } & 492 & 225 / 267 & 59.41 & 126 & 1694 & 15824 \\ \text { LUSC } & 489 & 362 / 127 & 65.32 & 176 & 1492 & 16748 \\ \text { OV } & 294 & 0 / 294 & 67.23 & 169 & 2224 & 16942 \\ \text { PAAD } & 175 & 96 / 79 & 59.19 & 42 & \text { NA } & 16893 \\ \text { READ } & 159 & 88 / 71 & 64.37 & 92 & 607 & 17177 \\ \text { SARC } & 259 & 118 / 141 & 64.58 & 22 & \text { NA } & 16472 \\ \text { SKCM } & 459 & 284 / 175 & 60.71 & 98 & 1991 & 16197 \\ \text { STAD } & 379 & 247 / 132 & 58.14 & 215 & 2454 & 16030 \\ \text { UCEC } & 541 & 0 / 541 & 65.49 & 146 & 1043 & 16885 \\ & & & 63.95 & 90 & \text { NA } & 16670\end{array}$




\section{Table 2 (on next page)}

Characteristics of the Tier 3 miRNA datasets in OncoLnc

Age at diagnosis is in years, and is an average. The events indicate the number of deaths in the dataset. Median survival is in days and could not be calculated for COAD, KIRP, READ, and UCEC. 


$\begin{array}{lrlrrrr} & & & & & & \text { Genes } \\ \text { Cancer } & \text { Patients } & \text { Male/Female } & \begin{array}{r}\text { Age at } \\ \text { Diagnosis }\end{array} & \text { Events } & \begin{array}{r}\text { Median } \\ \text { Survival }\end{array} & \begin{array}{c}\text { incoLnc } \\ \text { BLCA }\end{array} \\ \text { BRCA } & 904 & 297 / 107 & 68.02 & 177 & 1036 & 512 \\ \text { CESC } & 267 & 11 / 977 & 58.35 & 131 & 3941 & 479 \\ \text { COAD } & 426 & 226 / 200 & 48.27 & 59 & 4086 & 501 \\ \text { ESCA } & 144 & 125 / 19 & 66.48 & 84 & \text { NA } & 476 \\ \text { GBM } & 561 & 343 / 218 & 60.61 & 59 & 801 & 494 \\ \text { HNSC } & 501 & 363 / 138 & 57.94 & 67 & 2648 & 507 \\ \text { KIRC } & 506 & 331 / 175 & 61.30 & 208 & 1732 & 514 \\ \text { KIRP } & 286 & 210 / 76 & 60.48 & 165 & 2764 & 448 \\ \text { LAML } & 164 & 88 / 76 & 54.52 & 44 & \text { NA } & 430 \\ \text { LGG } & 506 & 278 / 228 & 43.07 & 100 & 518 & 374 \\ \text { LIHC } & 362 & 248 / 114 & 59.41 & 125 & 2660 & 486 \\ \text { LUAD } & 490 & 226 / 264 & 65.35 & 175 & 1492 & 495 \\ \text { LUSC } & 467 & 346 / 121 & 67.43 & 160 & 2224 & 519 \\ \text { OV } & 470 & 0 / 470 & 59.85 & 92 & 3128 & 467 \\ \text { PAAD } & 175 & 96 / 79 & 64.37 & 92 & 607 & 494 \\ \text { READ } & 154 & 84 / 70 & 64.23 & 22 & \text { NA } & 495 \\ \text { SARC } & 259 & 119 / 140 & 60.85 & 98 & 1991 & 455 \\ \text { SKCM } & 438 & 271 / 167 & 58.01 & 207 & 2470 & 535 \\ \text { STAD } & 400 & 260 / 140 & 65.54 & 155 & 1043 & 495 \\ \text { UCEC } & 534 & 0 / 534 & 63.91 & 87 & \text { NA } & 518\end{array}$




\section{Table 3(on next page)}

Characteristics of the MiTranscriptome beta analyses in OncoLnc

Age at diagnosis is in years, and is an average. The events indicate the number of deaths in the dataset. Median survival is in days and could not be calculated for COAD, KIRP, and UCEC. 


$\begin{array}{lrlrrrr} & & & & & & \text { Genes } \\ \text { Cancer } & \text { Patients } & \text { Male/Female } & \begin{array}{c}\text { Age at } \\ \text { Diagnosis }\end{array} & \text { Events } & \begin{array}{r}\text { Median } \\ \text { Survival }\end{array} & \begin{array}{c}\text { in } \\ \text { Oncolnc }\end{array} \\ \text { BLCA } & 120 & 86 / 34 & 67.37 & 61 & 706 & 4322 \\ \text { BRCA } & 766 & 8 / 758 & 58.03 & 111 & 3941 & 4708 \\ \text { CESC } & 106 & 0 / 106 & 48.22 & 26 & 3046 & 4493 \\ \text { COAD } & 117 & 52 / 65 & 69.64 & 24 & \text { NA } & 3302 \\ \text { GBM } & 144 & 94 / 50 & 59.56 & 24 & 1426 & 4524 \\ \text { HNSC } & 288 & 211 / 77 & 61.40 & 133 & 1762 & 4314 \\ \text { KIRC } & 457 & 299 / 158 & 60.75 & 156 & 2764 & 5191 \\ \text { KIRP } & 73 & 51 / 22 & 59.78 & 17 & \text { NA } & 4627 \\ \text { LAML } & 20 & 15 / 5 & 54.75 & 10 & 580 & 3940 \\ \text { LGG } & 217 & 123 / 94 & 42.82 & 65 & 2660 & 4875 \\ \text { LIHC } & 65 & 40 / 25 & 60.97 & 41 & 1005 & 3610 \\ \text { LUAD } & 320 & 148 / 172 & 65.72 & 118 & 1357 & 4636 \\ \text { LUSC } & 330 & 244 / 86 & 67.16 & 112 & 2284 & 4979 \\ \text { OV } & 369 & 0 / 369 & 59.63 & 69 & 3128 & 4901 \\ \text { READ } & 42 & 22 / 20 & 66.67 & 8 & 1581 & 3310 \\ \text { SKCM } & 255 & 159 / 96 & 56.82 & 148 & 2192 & 3893 \\ \text { STAD } & 148 & 93 / 55 & 65.72 & 56 & 940 & 4619 \\ \text { UCEC } & 274 & 0 / 274 & 63.12 & 39 & \text { NA } & 3706\end{array}$


1

Example of OncoLnc search results

The Cox coefficient and $p$-value are from the gene term in precomputed multivariate Cox regressions. The FDR correction is performed per cancer analysis per data type, and in this example the correction would have involved around 16,000 genes for each cancer. The rank is also performed per cancer per data type. In this example DONSON is the 3rd most highly correlated gene in KIRC.

\section{Cox regression results for DONSON}

\begin{tabular}{|c|c|c|c|c|c|c|c|}
\hline Cancer ${ }^{\mathbf{B}}$ & $\begin{array}{c}\text { Cox } \\
\text { Coefficient }\end{array}$ & P-Value & $\begin{array}{c}\text { FDR } \\
\text { Corrected }\end{array}$ & Rank ${ }^{(1)}$ & $\stackrel{\text { Median }}{\text { Expression }} \mathbf{1}$ & $\begin{array}{c}\text { Mean } \\
\text { Expression }\end{array}$ & Plot Kaplan? \\
\hline BLCA & -0.005 & $9.50 \mathrm{e}-01$ & $9.79 \mathrm{e}-01$ & 15844 & 410.38 & 478.94 & Yes Please! \\
\hline BRCA & 0.192 & $2.80 \mathrm{e}-02$ & $3.08 \mathrm{e}-01$ & 1494 & 268.08 & 341.28 & Yes Please! \\
\hline CESC & -0.152 & $2.50 \mathrm{e}-01$ & $5.95 \mathrm{e}-01$ & 6770 & 665.25 & 681.12 & Yes Please! \\
\hline COAD & -0.123 & $2.40 \mathrm{e}-01$ & $5.44 \mathrm{e}-01$ & 7106 & 279.33 & 296.04 & Yes Please! \\
\hline ESCA & -0.123 & $3.60 \mathrm{e}-01$ & $9.77 \mathrm{e}-01$ & 6053 & 334.31 & 379.24 & Yes Please! \\
\hline GBM & -0.224 & $3.20 \mathrm{e}-01$ & $9.62 \mathrm{e}-01$ & 5406 & 352.85 & 389.29 & Yes Please! \\
\hline HNSC & 0.009 & $9.00 \mathrm{e}-01$ & $9.64 \mathrm{e}-01$ & 15462 & 275.38 & 330.77 & Yes Please! \\
\hline KIRC & 0.574 & $8.00 \mathrm{e}-12$ & $4.45 \mathrm{e}-08$ & 3 & 148.77 & 169.63 & Yes Please! \\
\hline KIRP & 0.588 & $9.90 \mathrm{e}-05$ & $1.98 \mathrm{e}-03$ & 813 & 187.15 & 209.66 & Yes Please! \\
\hline LAML & 0.234 & $5.50 \mathrm{e}-02$ & $4.05 \mathrm{e}-01$ & 2051 & 381.27 & 401.45 & Yes Please! \\
\hline LGG & 0.333 & 4.10e-04 & $1.60 \mathrm{e}-03$ & 4308 & 203.47 & 230.77 & Yes Please! \\
\hline LIHC & 0.188 & $5.50 \mathrm{e}-02$ & $2.32 \mathrm{e}-01$ & 3738 & 300.81 & 354.61 & Yes Please! \\
\hline LUAD & 0.159 & $2.30 \mathrm{e}-02$ & $1.29 \mathrm{e}-01$ & 2946 & 253.81 & 295.14 & Yes Please! \\
\hline LUSC & -0.09 & $2.40 \mathrm{e}-01$ & $8.35 \mathrm{e}-01$ & 4762 & 329.73 & 380.92 & Yes Please! \\
\hline ov & -0.028 & $8.50 \mathrm{e}-01$ & $9.92 \mathrm{e}-01$ & 14385 & 253.27 & 303.44 & Yes Please! \\
\hline PAAD & 0.05 & $6.40 \mathrm{e}-01$ & $7.93 \mathrm{e}-01$ & 13828 & 187.31 & 196.42 & Yes Pleasel \\
\hline READ & -0.168 & $4.30 \mathrm{e}-01$ & $9.24 \mathrm{e}-01$ & 7545 & 269.13 & 278.58 & Yes Pleasel \\
\hline SARC & 0.255 & $2.20 \mathrm{e}-02$ & $1.54 \mathrm{e}-01$ & 2273 & 391.13 & 470.87 & Yes Please! \\
\hline SKCM & 0.146 & $3.80 \mathrm{e}-02$ & $1.51 \mathrm{e}-01$ & 3999 & 348.03 & 375.7 & Yes Please! \\
\hline STAD & -0.059 & $4.90 \mathrm{e}-01$ & $8.02 \mathrm{e}-01$ & 10245 & 304.86 & 327.4 & Yes Please! \\
\hline UCEC & 0.009 & $9.30 \mathrm{e}-01$ & $9.95 \mathrm{e}-01$ & 15404 & 278.83 & 315.72 & Yes Please! \\
\hline
\end{tabular}


2

Example of OncoLnc Kaplan-Meier results

A) Submitting non-overlapping percentiles will return a logrank $p$-value for the analysis and a PNG image with the option to generate a PDF of the plot.B) Below the Kaplan-Meier image will be the data that was plotted along with an option to download a csv file. 
a

\section{Kaplan plot for DONSON in KIRC \\ 25 \\ Submit}

If you make multiple plots be sure to reload the page to ensure an updated image!

\section{Go to PDF}

Logrank p-value $=2.07 \mathrm{e}-13$

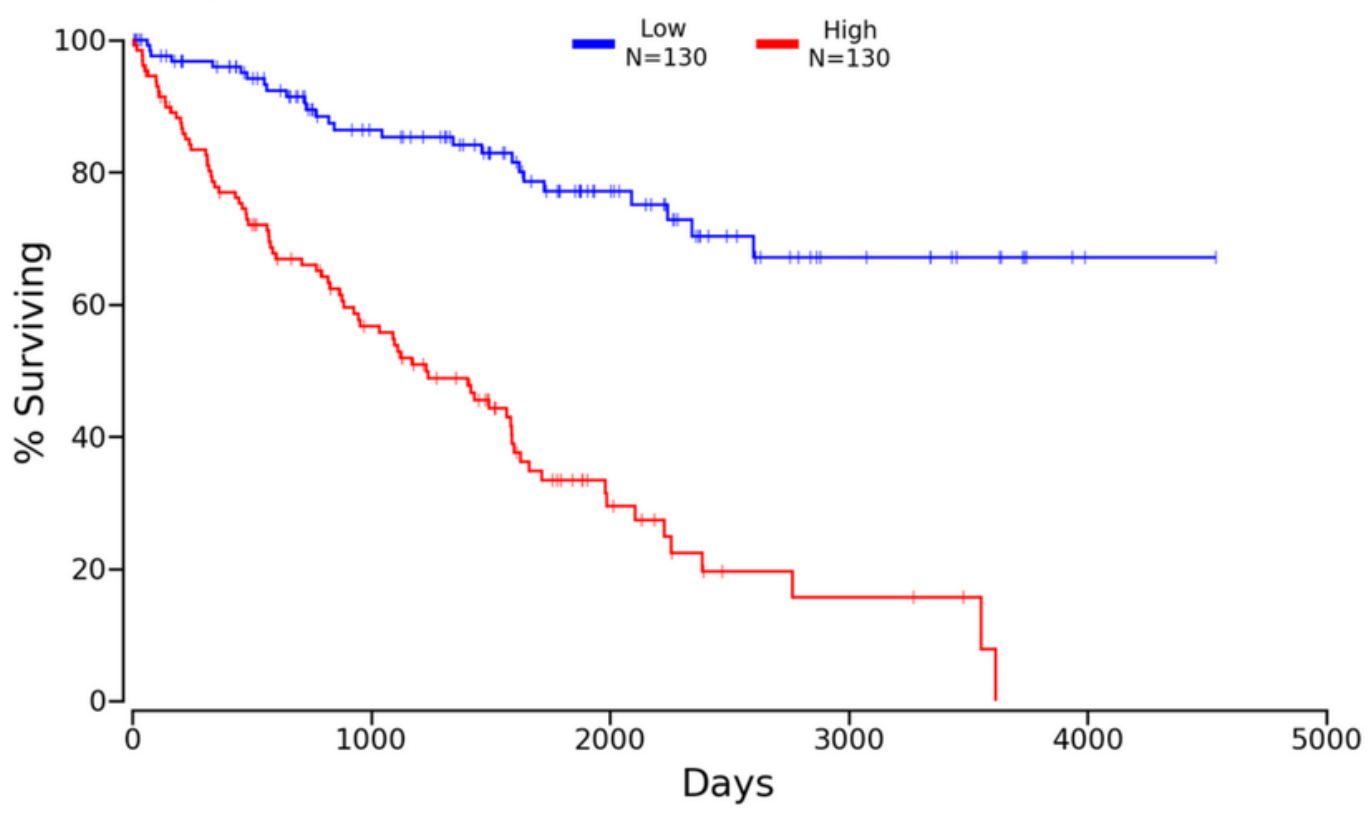

b

For an excel file of this data

\begin{tabular}{|c|c|c|c|c|c|c|c|c|}
\hline Patient & Days & Status & Expression & & Patient & Days & Status & Expression \\
\hline TCGA-BO-5702 & 2172 & Alive & 15.78 & & TCGA-B8-5163 & 822 & Alive & 205.01 \\
\hline TCGA-AK-3447 & 1217 & Alive & 37.58 & & TCGA-CJ-4641 & 1661 & Dead & 205.13 \\
\hline TCGA-B0-5117 & 1608 & Alive & 44.0 & & TCGA-B 0-5116 & 1274 & Alive & 205.5 \\
\hline TCGA-A3-3374 & 1314 & Alive & 47.14 & & TCGA-BP-5010 & 878 & Dead & 205.98 \\
\hline TCGA-BO-4834 & 2090 & Dead & 47.37 & & TCGA-B8-A54G & 53 & Alive & 206.17 \\
\hline TCGA-AK-3453 & 2531 & Alive & 47.92 & & TCGA-BP-4981 & 1097 & Dead & 206.24 \\
\hline TCGA-B2-5636 & 919 & Alive & 50.59 & & TCGA-CW-6087 & 41 & Dead & 206.47 \\
\hline TCGA-B0-5083 & 1045 & Dead & 50.85 & & TCGA-BP-4986 & 785 & Alive & 206.54 \\
\hline TCGA-CW-5587 & 2226 & Alive & 51.64 & & TCGA-BP-4771 & 162 & Dead & 207.08 \\
\hline TCGA-BP-4177 & 1670 & Alive & 54.22 & & TCGA-A3-3382 & 574 & Alive & 207.49 \\
\hline TCGA-B8-5553 & 435 & Alive & 54.85 & & TCGA-BP-4774 & 1885 & Alive & 207.69 \\
\hline TCGA-B8-4619 & 523 & Alive & 55.0 & & TCGA-CW-6097 & 571 & Dead & 208.62 \\
\hline TCGA-BO-5402 & 1290 & Alive & 55.77 & & TCGA-CZ-4862 & 3271 & Alive & 208.64 \\
\hline TCGA-AK-3428 & 3728 & Alive & 56.53 & & TCGA-B0-5081 & 362 & Dead & 208.65 \\
\hline TCGA-AK-3440 & 2865 & Alive & 56.99 & & TCGA-CJ-4888 & 1567 & Dead & 208.69 \\
\hline TCGA-B0-5695 & 2150 & Alive & 60.74 & & TCGA-CZ-5989 & 1905 & Alive & 209.11 \\
\hline TCGA-BP-4801 & 1124 & Alive & 60.78 & & TCGA-BO-5094 & 333 & Dead & 209.96 \\
\hline TCGA-CW- 5585 & 2609 & Alive & 61.84 & & TCGA-BO-4836 & 1238 & Dead & 212.42 \\
\hline TCGA-B2-3923 & 992 & Alive & 62.04 & & TCGA-CJ -4640 & 3480 & Alive & 212.94 \\
\hline TCGA-BP-4994 & 1308 & Alive & 62.19 & & TCGA-B 0-4706 & 65 & Dead & 214.73 \\
\hline TCGA-CZ-5451 & 1929 & Alive & 63.53 & & TCGA-A3-3380 & 567 & Alive & 215.69 \\
\hline TCGA-BP-5168 & 1463 & Dead & 63.89 & & TCGA-CZ-4861 & 446 & Dead & 216.26 \\
\hline TCGA-BP-5192 & 714 & Alive & 63.99 & & TCGA-BO-5102 & 2764 & Dead & 216.52 \\
\hline TCGA-CW- 5583 & 2489 & Alive & 64.31 & & TCGA-A3-3322 & 1478 & Alive & 217.26 \\
\hline TCGA-CJ-4885 & 3451 & Alive & 64.64 & & TCGA-A3-3325 & 1170 & Dead & 218.45 \\
\hline TCGA-BO-5705 & 4537 & Alive & 65.52 & & TCGA-BP-4989 & 118 & Alive & 219.2 \\
\hline TCGA-AK-3450 & 1779 & Alive & 65.86 & & TCGA-BP-4346 & 1493 & Dead & 219.38 \\
\hline
\end{tabular}

